\title{
Modelagem Computacional do Crescimento do Glioma via Diferenças finitas
}

\author{
Júnior J. da Silva* Vanessa S. Garcia ${ }^{\dagger}$ \\ Modelagem Computacional em Ciência e Tecnologia, EEIMVR, UFF, \\ 27255-125, Volta Redonda, RJ \\ E-mail: junior-jds@oi.com.br, vanessa.sgarcia@gmail.com, \\ Gustavo B. Alvarez Diomar C. Lobão \\ Departamento de Ciências Exatas, EEIMVR, UFF, \\ 27255-125, Volta Redonda, RJ \\ E-mail: benitez.gustavo@gmail.com, lobaodiomarcesar@yahoo.ca.
}

\section{RESUMO}

Um dos fenômenos mais agressivos, o câncer, tem despertado a pesquisa de inúmeros modelos matemáticos capazes de descrever a evolução desta neoplasia e ajudar, desta forma, no entendimento da doença e na aplicação das terapias existentes. Em particular, o glioma de grau IV, tumor cerebral primário, possui uma combinação de rápido crescimento e invasibilidade refletindo na taxa de $100 \%$ de fatalidade, se tornando um campo de pesquisas para novas abordagens terapêuticas e plannejamento das terapias convencionais.

A simulação destes modelos matemáticos nos permite compreender a evolução do tumor em resposta à terapia sem a necessidade de expor a vida do paciente ao risco, por se tratar de uma técnica não invasiva. Dentre os modelos existentes, Bellomo et al. [1] descreve o modelo de crescimento de células de glioma proposto por Swanson et al. [2] a qual simulou, utilizando o método numérico de elementos finitos de Skeel e Berzins, para alguns tipos de fracionamentos de dose descrito em [2]. O modelo é descrito por uma equação do tipo reativa difusiva com condição de contorno de Neumann, por não ocorrer metástase para este tipo de tumor, como segue:

$$
\frac{\partial c}{\partial t}=\nabla \cdot(D \nabla c)+\rho c-R(\alpha, d(x, t)) c,
$$

onde $d=d(x, t)$ representa a dose fracionada definida no espaço e tempo, $c=c(x, t)$ é a concentração de células tumorais no domínio, $D$ o coeficiente de difusão e $\rho$ a taxa de proliferação. O termo que descreve os efeitos da radioterapia é dado do seguinte modo:

$$
S(\alpha, d(x, t))=e^{-\alpha B E D}, \quad B E D=N\left(d+\frac{d^{2}}{\alpha / \beta}\right),
$$

e

$$
R(\alpha, d(x, t))= \begin{cases}0, & \text { para } \mathrm{t} \in \text { terapia } \\ 1-S(\alpha, d(x, t)), & \text { para } \mathrm{t} \notin \text { terapia }\end{cases}
$$

\footnotetext{
*bolsista de mestrado Capes

†bolsista de pós-doutorado Capes
} 
Este termo relaciona o modelo linear quadrático, que calcula os efeitos causados pelo fracionamento de dose, com a dose biológica efetiva $(B E D)$ para calcular a probabilidade de sobrevivência das células $(S)$. O número de frações da dose é dado por $N$, já $\alpha$ e $\beta$ são os parâmetros ajustáveis do modelo linear quadrático [1].

O propósito deste artigo é simular o crescimento de células tumorais (glioma) em resposta à radioterapia a partir do modelo de Swanson et al. [2] para 5 esquemas de tratamento abordados em [2], utilizando o método de diferenças finitas, especificamente o método de Crank-Nicolson, fazer a análise de estabilidade do método e comparar de forma quantitativa os resultados obtidos neste trabalho com os de Swanson et al. [2].

A simulação do modelo (1) foi feita para o caso unidimensional, através do método de CrankNicolson [3] e implementada em MatLab, utilizando as mesmas condições iniciais e esquemas de terapias descrito em [2]. Os resultados obtidos se encontram na Figura 1, a qual descreve a evolução do raio do tumor de acordo com os dias e os esquemas de tratamento.

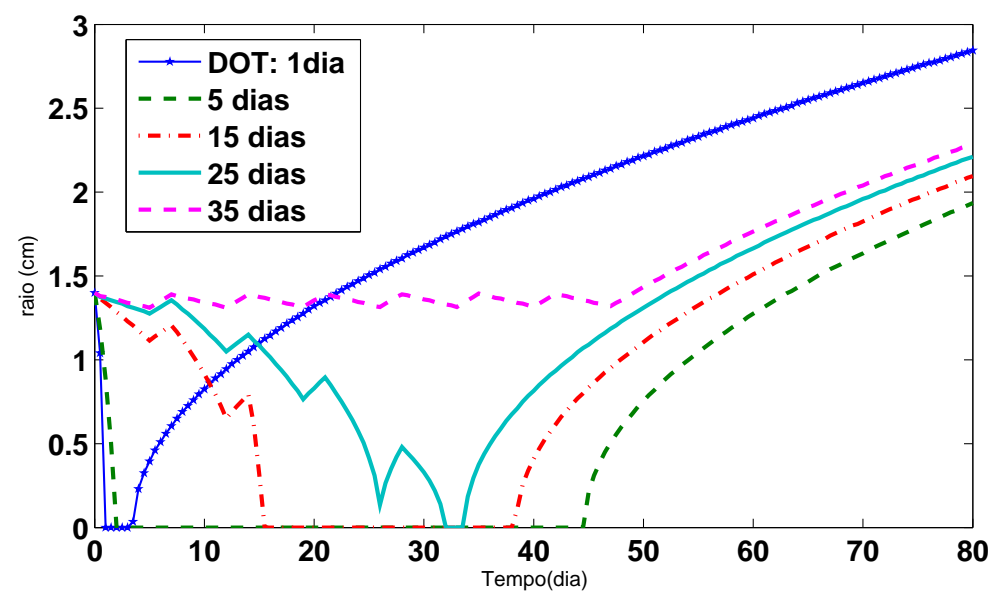

Figura 1: Crescimento do raio do tumor em resposta à radioterapia

A análise de estabilade para o método de Crank-Nicolson neste problema nos mostra que o método é instável, podendo apresentar oscilações mesmo que pequenas. A comparação dos resultados obtidos neste trabalo com os de Swanson et al. [2] mostra que nos casos simulados por nós a solução é mais suave, apresentam oscilações menores, porém a malha foi mais refinada, já no caso de Swanson et al. [2] apresenta oscilações mais percepitíveis, durante toda a simulação. Em ambos os casos os resultados são próximos e a presença de oscilações, ocasionada pela instabilidade numérica, mostra a necessidade de desenvolver novas estratégias, que gerem resultados mais confiáveis e precisos, principalmente para simulações em mais de uma dimensão espacial.

Palavras-chave: Crescimento de Células Tumorais, Método de Crank-Nicolson, Equação da Difusão Reação, Glioma

\section{Referências}

[1] N. Bellomo, M. Chaplain, E. Angelis, "Selected Topics in Cancer Modeling: Genesis,Evolution,Immune Competition and Therapy", Birkhäuser, 2008.

[2] R. Rockne, E. C. Alvord Jr, J. K. Rockhill, K. R. Swanson, A mathematical model for brain tumor response to radiation therapy, Journal of Mathematical Biology, 58(2008), 561-578.

[3] J. C. Tannehill, D. A. Anderson, R. H. Pletcher, "Computational Fluid Mechanics and Heat Transfer", Taylor and Francis,1997. 